\title{
The Study and Optimization of CDMA Network Pilot Pollution
}

\author{
Qi LIU ${ }^{1, a}$, Zhi Jian YIN ${ }^{2, b *}$ and Zhao Pan WU ${ }^{3, c}$ \\ 1. 2. 3 Jiangxi Science and Technology Normal University, Nanchang JiangxiProvince, China \\ a20423232@qq.com, b86412656@qq.com *Corresponding author, '654591305@qq.com
}

Keywords: drive testing , pilot pollution, network optimization

\begin{abstract}
This paper focuses on studying and analysing the Pilot Pioneer drive testing system's the pilot pollution problem, designing and putting forward quantitative analysis and the algorithm with absolute difference on the process of diagnostic analysis, It can effectively locate the pilot pollution area by this analysis method. we can see from the result of network optimization case studying, the method of diagnostic module quantitative analysis can effectively detect the pilot pollution problem that existing in wireless network, it can also effectively locate the occurred area, doting on the geographic view, Compared with the traditional analysis method, this method can efficiently reduce the scope of the problem area, and be equipped with the higher problems positioning accuracy.
\end{abstract}

In the CDMA2000 network program, the system adopts the same frequency networking mode called self-interference, which making CDMA system pilot pollution issues outstanding, especially high-rise buildings having gradually replaced most of the old multi-story buildings, which having added the wireless signal reflection and refraction effects.

\section{Introduction}

\subsection{The definition of pilot pollution}

When the concentrated presence of the mobile station (MS) of the activation of multiple signal strength is greater than a certain threshold of the pilot signal, similar to the signal strength of the pilot signal or less, and the number of pilot isn't less than four, then the mobile station can' $t$ discern a leading pilot signal, resulting in dropped calls, etc.. This phenomenon is known as pilot pollution.

In CDMA2000 network, it can be considered that the area exists pilot pollution when there are more than three useful signals and the similar pilot strength of the main serving cell appear at the same position.

Quantified definition pilot pollution: strong pilot signal is defined as Rx Power $>=-95 \mathrm{~dB}, \mathrm{Ec} / \mathrm{Io}>$ $=-12 \mathrm{~dB}$ useful signal; Too much strong pilot signal refer to the number of pilot signals is less than four, If the difference between the first and the fourth pilot signal is more than $2 \mathrm{~dB}$, which can be defined there is strong enough dominate pilot in the point.

\subsection{The cause of the pilot pollution}

The same frequency self-interference characteristic in CDMA2000 system and the complexity of signal propagation conditions make the contamination diversity of pilot pollution in CDMA2000. The occurrence of Pilot pollution is not necessarily caused by only one certain reason, it may be caused by composite factors. Generally speaking, it is considered by the inappropriate location of base stations, unreasonable layout of community, the impact of surrounding geographical environment, the effect of high-rise buildings and the antenna azimuth angle' $\mathrm{s}$ wrong placement, the position error of antenna height, which are the general factors that causing pilot pollution.

\subsection{Pilot Pollution Module Algorithm Design}

In practice, checking the pilot pollution inspectors is usually using the driving test software, this paper collects the data through Pilot Pineer, and then uses the diagnostic analysis of driving test analysis software on those collected data. Development and pollution area network optimization solutions implementation depend on the results of the analysis.

Algorithm 1

In the transmission and reception meet the condition that Tx Power $<=15 \mathrm{dBm}$ and at the premise $\mathrm{Rx}$ Power $>=-90 \mathrm{dBm}$, when the pilot numbers that Ec / Io is more than $-12 \mathrm{~dB}$ greater than 
the threshold, it means that the presence of pilot pollution, the setting of initial numeric is Ec / Io of Set to -12 , the pilot difference setting is $1 \mathrm{stEc} /$ Io and 4thEc / Io of the difference is 0-2 .in CDMA2000 1x and EVDO system, the threshold defaults 2. The number of pilot threshold should be set at least four, Limit Pilot Count initial numeric defaults 3.

Algorithm 2

The algorithm checks Ec / Io through T_ADD, firstly, setting the active set threshold that T_ADD, when the position of the mobile station is located on more than three pilot of Ec / Io of not less than T_ADD, and the pilot difference is less than or equal to 2, Then the fourth pilot is the pollution sources of the first pilot, which producing the phenomenon of pilot pollution. The difference is generally set as a positive number, the default initial numeric of Pilot Count is Limited the Pilot Count at 3.

\section{The detection of pilot pollution problem}

\subsection{Case description}

Road testing near Linyi University of the urban district, At the western part of Shuangling Road near the South Gate of Linyi University, when the test vehicle was traveling from east to west, during the time period of 11:30:41:139, Ec / Io and Rx are normal, but obviously FFER increased, Ec / Io and Rx suddenly started to fall at some sections. The base station' $\mathrm{s}$ distribution of this section is shown in Figure 1.

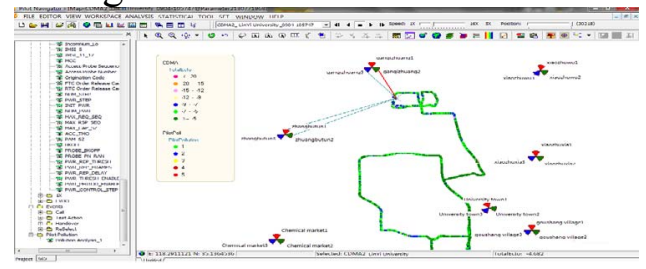

Figure 1: The base stations' Distribution

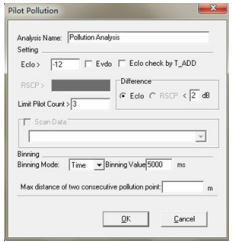

Figure 2 pilot pollution setting

The reason Analysis of this phenomenon

(1) Check the alarm information at this station, no alarm;(2) check the antenna feedback information, normal;(3) Check the adjacent area' $s$ configuration of the base stations, without omission adjacent area, the adjacent area is normal priority;

From the above data, we can judge that this area exists pilot pollution or pilot interference.

\section{2 pilot pollution testing and certification}

By Dingli Pilot Navigator road test software, using the pilot pollution detection of the diagnostic and analysis setting, the steps of the pilot pollution detection are as following:

(1) Testing data and importing base data

Firstly, opening the data file under the editor section of the navigation column, concrete steps are "Edit" - "Data File" - "Import." , opening the test data and importing to the window.After the importing completely and selecting loading maps, In order to more accurately locate the position in the process of analysing, and it can directly reflect the geographic surroundings .

(2) The pilot pollution' s parameter setting

Firstly, setting an Ec / Io threshold, selecting the number of the pilots defaulted of pilot pollution(Limit Pilot Count), setting a pilot absolute difference, then Bin analysis the type and data , conducting the pilot pollution analysis by analyzing the mean after Ec / Io and the Ec / IoBin reference. The interface setting is shown in Figure 2.

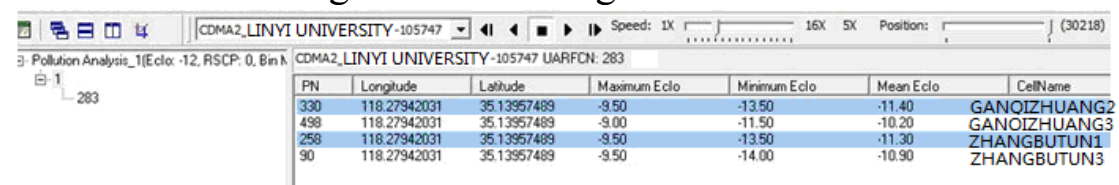

Figure 3: pilot pollution point table view

(3) Conducting the pilot pollution analysis, generating the pilot pollution view (table view and geographic view). The table view of the pilot pollution spot as shown in Fig.ure 3 . 
Through the result of view, we can diagnose and analyze the pilot pollution according to the principle of pilot pollution. The pilot pollution view can clearly indicate the spot' $\mathrm{s}$ intensity pollution of the pilot pollution. The position of pilot pollution is shown in Figure 4 .

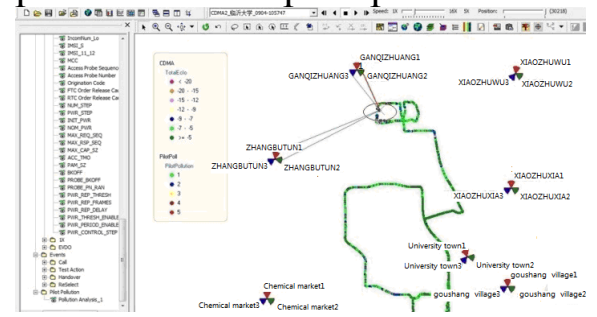

Figure 4 : pilot pollution point

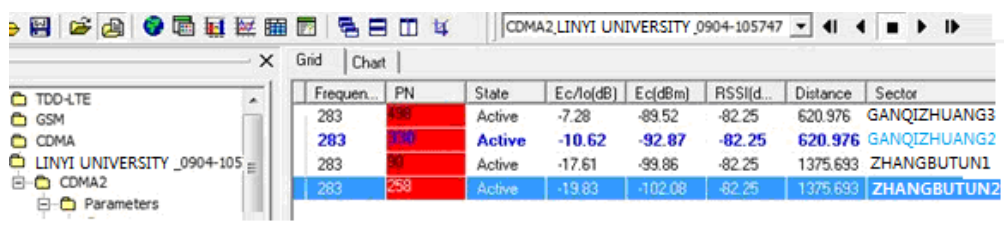

Figure 5: the neighbor community' parameter list before optimization

\section{The analysis}

Pilot pollution point appears near the interchange of the double Ridge Road' s west section and Binhe Avenue, the south of this region is the high-rise buildings, the north side is near the Beng river, the east side is the exhibition area of the development Garden Fair, due to the reflection of high building and river ref, it causes the FFER' increasing of this area, and leads to increase the rate of the calls connecting and the call' s quality. The neighbor community parameter list before optimization is shown in Figure 5.

\subsection{The effects of pilot pollution}

Effect of the pilot pollution on network' s performance at following aspects:

(1) Increased the rate of calling failure and the switching failure, lead to calls dropped.

First, the generation of calls failure that each mobile station in the active set pilot strength Ec / Io or less pilot pollution in the area, resulting in MS can' $\mathrm{t}$ choose the main pilot. Lead to the calls' failing. Secondly, the problem is due to switch failure when the MS is on the moving, MS can not search for the sufficiently strong pilot, but still keep the frequent switching state, but also affect the system capacity, causing the system' s load increasing. Finally, if the MS is calling at this time, to enter the pilot pollution area, since the frequently switching problem or the new system of strong activation of the pilot are not set, may cause the calls dropping.

(2) The calls failure rate' $s$ improvement of the High-speed data business

Typically, high-speed data services require a larger Ec / lo values, and more stable wireless environment. As the pilot pollution occurred, it is difficult to find a stable frequency to the maximum intensity of the dominant signal, which is not very conducive to high-speed data call access.

\subsection{Solutions to Pilot Pollution}

In actual network optimization of CDMA2000 network, pilot pollution of solutions, According to the different cause, the solutions to Pilot Pollution should adopt the different embodiments. The key lies in becoming a dominant frequency in the pilot pollution area.

Several solutions are given that based on the causes of the pilot pollution :

1. The adjustment of the base station' s antenna

Adjusting the antenna of the base station' s height and the up or down azimuth angle, because the cascade affect is small, so that is the preferred solution of pilot pollution problem, the fundamental principle and objective just is to increase the dominant frequency by adjusting the base station antennas.Considering the Linyi Telecom, The antenna used in actual network is still the traditional height of 35 meters. Lanshan of the Linyi City generally choose to install the antennas that the electronic inclination angle of about 6 degrees.

2. Adjust cell base station transmission power

The goal of the base station transmit power adjustment is to generate the dominant frequency, the focus of power adjustment is to open close Ec / Io gap from the power aspect, so as to improve the pilot pollution.

3. Open the new station, built repeater or chamber subsystem

Adding a new station or repeater approach is generally used for large traffic area, if the area is obvious pilot pollution, we can take this approach. The construction of indoor distribution system is 
major to improve indoor coverage problems. Constructing the indoor distribution system in high-rise buildings is a way of solving the problem of high-level pilot pollution. Because of the path loss and other reasons, the establishment of new cell signal will become the dominant frequency signal, which can effectively solve the pilot pollution problems in the region.

4. The adjustment of wireless parameter

The adjustment of system switching threshold include two kinds, One is to adjust the system switching thresholdthe, the other is the adjustment of the maximum allowable number of switching cell. Increasing system switching parameter thresholds reasonably can effectively control active set number of active pilots, which preventing pilot pollution.

5. Adjustment of the neighbor cell base station PN code

Under normal circumstances, the antenna transferring or controlling pilot to pilot has a certain interference power. When the above operation does not work, we can try to adjust the BTS PN code. It is because that the adjusted PN code is added to the queue Neighborhood, which interference pilot having become a useful signal.

\section{Optimization scheme}

Seeing from the pilot pollution 4, we can see that there are pilot pollution spot the latitude longitude 118.27942031 35.13957489, 283 frequency position, the difference of four adjacent cells and primary serving cell appears at this point Ec / Io is not big, After the bin treatment, the difference in the set is within a threshold range. Due to the strong disturbance leading in the region adjacent cell frequency dominant frequency, where the dominant frequency signals can not be formed.

After verification, pilot pollution point appears near Lanshan road and Binhe avenue area with double Ridge Road Interchange, south of the high-rise buildings, altar inside ancestral temple, north of the river, the east side of the development of Garden Expo exhibition, based on the data test, the base distribution and geographical environment, sector 1 Adjustable feet 2 port $v$ illage base station and the sector under pressure 1o proposed here can be adjusted. But taking the special geographical location of the region, the less number of peripheral base station and high traffic into account, adjusting the lower inclination husband port village base station may lead to the covering field strength problems, it is recommended to build new base and enhance dominant frequency, thus completely solving the problem of pollution in the region of the pilot.

The problem of the region pilot pollution has been solved after optimization.

\section{5 suggestion and summary}

For the optimization of the complex topography pilot pollution, we must consider the wireless surrounding environment and optimize the concept of clusters, so as to avoid the situation that solving one problem and bringing another new problem, at the same time, we also need to adjust the antenna, optimize adjacent areas and system parameters results.

\section{References}

[1] Niu Min. The Research and Implementation of CDMA network senior pilot pollution verification system. [MS Thesis]: Beijing: Beijing University of Post\$Telecommunication.2011

[2] Zhong WeiWei. The analysis and application of TD-SCDMA network optimization drive testing systems:.[MS Thesis]: Wuhan: Wuhan University of Technology .2009

[3] Zhao Qiang CDMA2000 1x EV-DO system. The interface and wireless network optimization. Beijing: People's Posts and Telecommunications Press, 2013

[4] Wang Xiaolong WCDMA signaling analysis and network optimization Beijing: Mechanical Industry Press, 2013

[5] Fei Min .The study of CDMA2000 network optimization. [MS Thesis]: Xi'an: Xidian University, 2010

[6] Dinglicom.Pilot Pioneer. Http://www.dinglicom.com/products. 2013 